\title{
Resource Sharing for Granted \& Grant-free Demands in 5G for Non-orthogonal Multiple Access
}

\author{
K.Raghavendra, N.Sreekanth, P.Swamy
}

\begin{abstract}
In the present days we observed a drastic change in the usage of mobile internet as well as advancements in Internet of things. With this advancements in mobile communications as well as IOT, the present generation mobile communication i.e. $4 G$ technology is considered to be not sufficient to reach to the needs of common people, therefore the upcoming generation $5 G$ needs in a frame way to face to ultra huge network capacity \& massive wireless connectivity. A major hopeful technology which has the capacity for enabling different user equipments, sharing interweaving wireless resources in $5 G$ is named as Non Orthogonal multiple Access (NOMA) technology, where most of the industrialists \& academicians are doing research in recent years To establish good Quality of Service $(Q o S) \&$ also to obtain low access latency as well as overhead signaling NOMA must compulsorily support two kinds of approaches: i) Scheduling approach ii)Random approach. The major challenge we observe in these two kinds of approach is how efficiently we obtain simultaneous support. Here we are proposing an approach for combining the granted \& grant free demands which works on the principle of allowing the approaches to share the same resources simultaneously. We are proposing heuristic resource algorithm which improve capacity of network \& user connectivity of NOMA in $5 G$ networking.
\end{abstract}

Keywords : Heuristic Resource, NOMA

\section{INTRODUCTION}

I In the recent years as many technologies evolved, using fourth generation for communication is very difficult to withstand the demands from the users. The key technology for communication is the fifth generation which is a revolutionary technology studied worldwide [1]. Any technology needs two most important features which are connectivity \& capacity [6]. Connectivity: As many technologies like vehicle to vehicle(V2V), Internet of things (IOT), Machine to Machine (M2M) \& Device to Device (D2D) needs a continuous connectivity which can be achieved using the fifth generation [10]. Capacity: As many features are added to the mobile internet like HD video access, Virtual Reality, Artificial Intelligence needs a ultra huge capacity which can be achieved using fifth generation[2, 11].

The basic idea about NOMA (Non-orthogonal multiple access) is to give access of resources to the users with overlap

\footnotetext{
Revised Manuscript Received on December 22, 2019.

K.Raghavendra ,Assistant Professor,Dept. of ECE, Mallareddy Engineering College for women, Secunderabad, raghu.siiet@gmail.com

Dr. N.Sreekanth,Professor, Dept. of ECE, Mallareddy Engineering College for women, Secunderabad, nsreeku@gmail.com

P.Swamy ,Assistant Professor, Dept.of ECE, TKREC,Ranga Reddy, swamygouds@gmail.com
}

spectrum which is attracting many researchers as the bandwidth can be achieved without any issues. A single spectrum can be used by many users, correspondingly to carry multiple resource information's. Many challenges need to address as the traditional multi access scheduling is done for uplink \& downlink by the base station. One of the most prominent issues when scheduling multi access is high access latency \& signaling overhead [3].

To overcome this issue along with scheduling access a random access is used to provide a prominent quality of service which is grant free access $\&$ granted access as one is used for periodical service $\&$ the other is for low latency [4]. Both the services are to be used to provide the hassle free communication [7]. This paper provides the information to use efficient multiple accesses with proper resource allocation developing a method which is to add granted \& grant free method called as semi grant method [5].

This article mainly concentrates on mixing or using same spectrum for both granted $\&$ grant free methods. It is also used for orthogonal multiple access such as OFDM \& MIMO [8]. The rest of this article is organized as section 2 for the principles \& applications of granted \& grant free access, section 3 for system model \& analysis \& finally section3 for summarized.

\section{Granted \& Grant Free Multi AcCess:}

Principle:

Assume that there are $\mathrm{P}$ sub carriers in a communication system, \& let $\mathrm{X}$ denotes granted access \& $\mathrm{Y}$ as Grant free access. $\mathrm{R}$ denotes available resources for granted access \& $\mathrm{S}$ as grant free.

$\forall \mathrm{R}=\varnothing, \mathrm{S} \neq \varnothing \rightarrow \mathrm{R} \cap \mathrm{S} \neq \varnothing$

Therefore this method is used for both granted access \& grant free access to share the same resources, by this technique first the granted access will take control by the system, \& after that grant free system collects the data which is gathered using random access. The base station first allocates that granted demands \& then as per the availability of uplink randomly grant free demands will be considered [9]. This is how granted \& grant free principle works in multi access environment.

\section{Application:}

Here we discuss about the uplink application cases where six users are considered where three are granted demands \& the remaining are grant free demands as shown in the below figure. 


\section{Resource Sharing for Granted \& Grant-free Demands in 5G for Non-orthogonal Multiple Access}

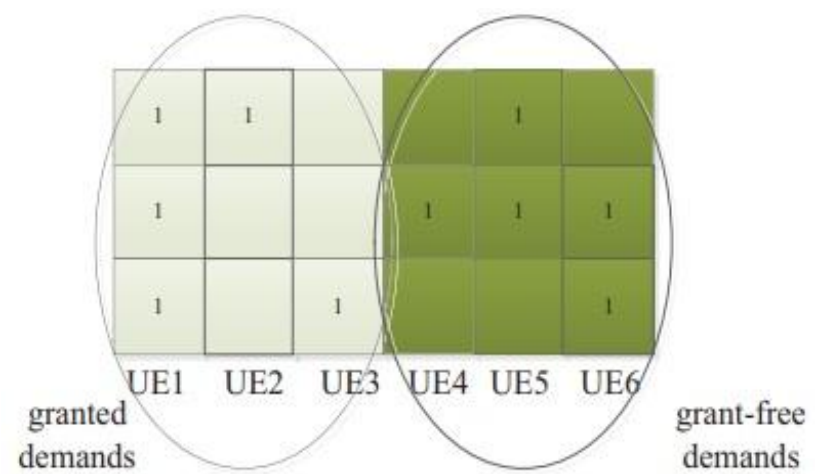

In this the base station allocates several columns for granted \& grant free demands where the channel is multi dimensional. The below diagram provides the information regarding the Time $\&$ frequency resources sharing for both granted \& grant free methods.

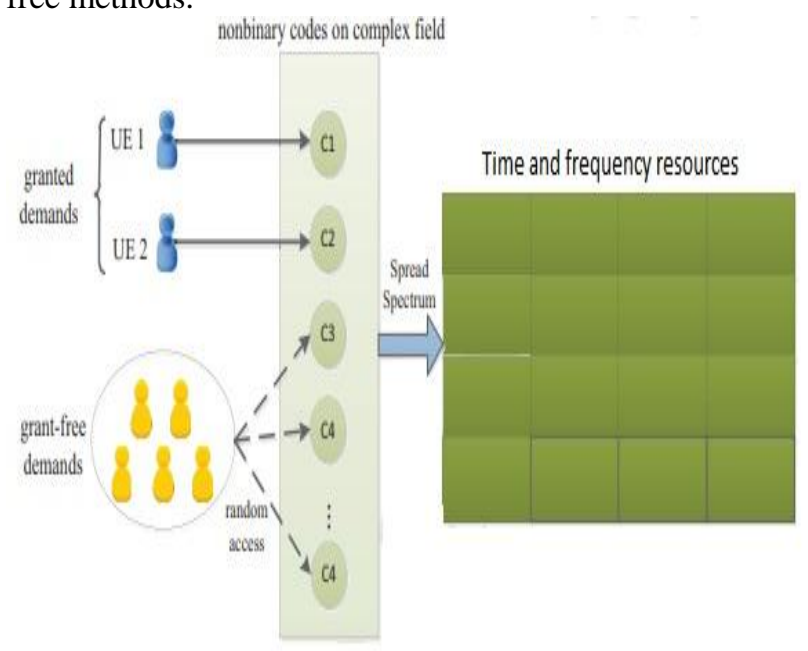

The base station splits the users using the non binary codes which are complex for granted access $\&$ random for grant free access.

\section{SYSTEM MODEL \& ANALYSIS}

\section{System model}

Every cell which is granted are located at geometric centre in the base station \& grant free are available at randomly distributed in a cell as shown in the below figure.

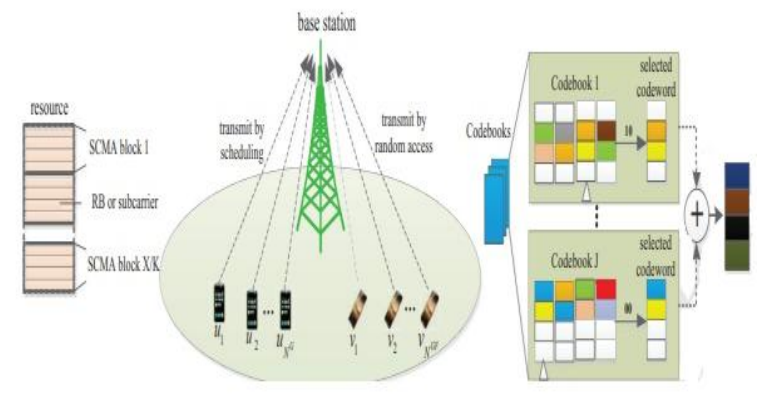

Let $\mathrm{U} \& \mathrm{~V}$ represent granted \& grant free demands which are represented as $\mathrm{U}=\left[\mathrm{U} 1, \mathrm{U} 2, \ldots . . . \mathrm{U}_{\mathrm{N}}\right] \& \mathrm{~V}=\left[\mathrm{V} 1, \mathrm{~V} 2, \ldots . . \mathrm{V}_{\mathrm{N}}\right]$, the scheduling is done using both random access \& fixed scheduling using heuristic resource algorithm. This algorithm provides the information regarding the channel availability $\&$ spectrum availability when the resources are to be shared.

\section{ANALYSIS}

The analysis is done for both granted \& grant free demands separately, in granted demands the base station has the complete knowledge about mapping for the cells along with the resource sharing. The signal interference \& noise ratio is calculated, in grant free demands the base station will not have any idea about the resource sharing, by this way the calculation for the noise ratio will be difficult. The SNR will be assumed in the case of grant free demands.

$\mathrm{C}^{\prime}=\mathrm{C}^{\mathrm{G}}+\mathrm{C}^{-\mathrm{GF}}$

$\mathrm{G}$ represents granted \& GF represent grant free demands. The capacity can be increased using the below formula $\mathrm{C}^{\prime}=\mathrm{C}^{\mathrm{G}}+\mathrm{C}^{-\mathrm{GF}}$

$$
\begin{aligned}
& \sum_{i=1}^{n-g} \sum_{j=1}^{j} T i_{s} j C G i_{s} j+p N-g f\left(1-\frac{1}{j}-n-g\right) p(N- \\
& G E-1) \cdot \int \log _{2}(1+x) \cdot f(x) \cdot d x
\end{aligned}
$$

\section{Working Principle of Heuristic Algorithm}

A Heuristic function takes a look at search algorithms. At each branching step, it assesses the available data \& takes a decision on which branch to follow. It does so by ranking substitutes. The Heuristic is any device that is often effective but will not guarantee work in every case.

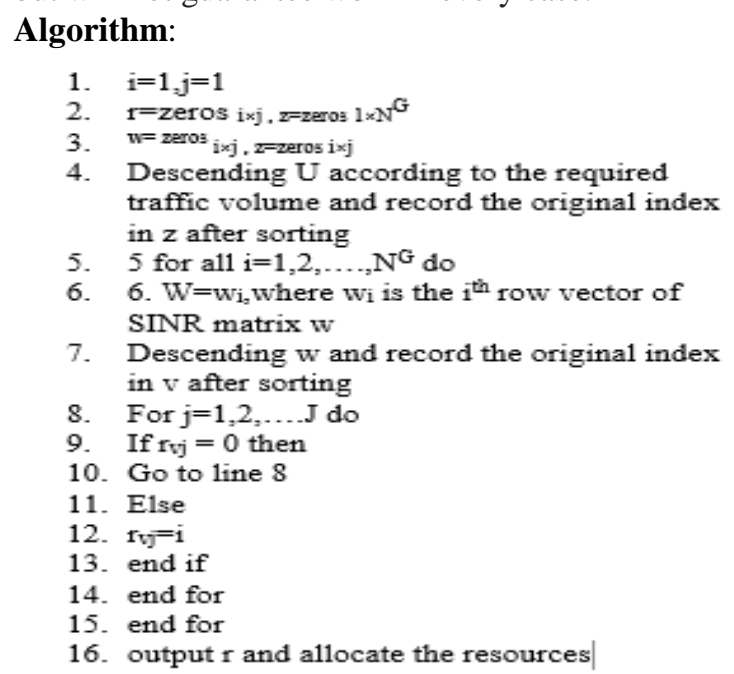

According to the algorithm the bit error rate is provided as follows

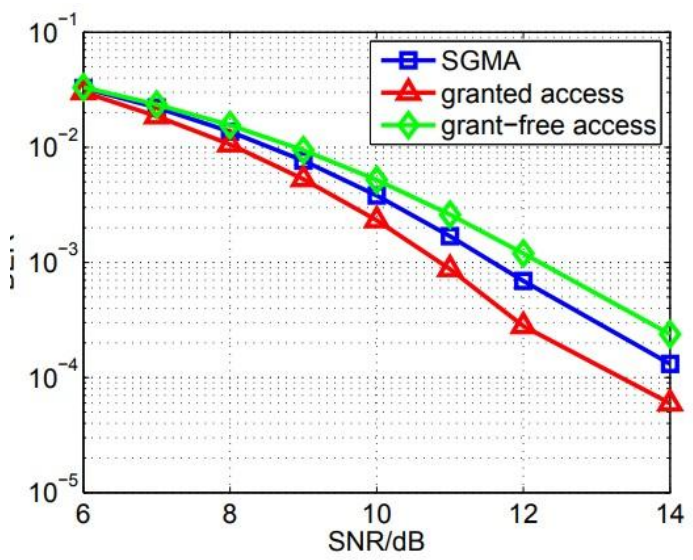




\section{CONCLUSION}

In this paper we introduced the granted $\&$ grant free demands sharing with the base station using the Heuristic algorithm which shows the importance of sharing the resources \& SNR with low spectrum utilization \& QoS. The article proposed the algorithm such that both the sequential \& random access is done with both granted \& grant free demands.

\section{REFERENCES:}

1. J. G. Andrews, S. Buzzi, W. Choi, S. V. Hanly, A. Lozano, A. C. K. Soong, J. C. Zhang, What will 5g be?, IEEE Journal on Selected Areas in Communications 32 (6) (2014) 1065-1082. doi:10.1109/JSAC.2014. 2328098.

2. M. Gholibeigi, N. Sarrionandia, M. Karimzadeh, M. Baratchi, H. van den Berg \& G. Heijenk, "Reliable vehicular broadcast using 5G device-to-device communication," 2017 10th IFIP Wireless \& Mobile Networking Conference (WMNC), Valencia, 2017, pp. 1-8.doi: 10.1109/WMNC.2017.8248846.

3. L. Dai, B. Wang, Y. Yuan, S. Han, C. 1. I, Z. Wang, Non-orthogonal multiple access for $5 \mathrm{~g}$ : solutions, challenges, opportunities, \& future 475 research trends, IEEE Communications Magazine 53 (9) (2015) 74-81. doi:10.1109/MCOM.2015.7263349

4. Z. Yuan, G. Yu, W. Li, Y. Yuan, X. Wang, J. Xu, Multi-user shared access for internet of things, in: 2016 IEEE 83rd Vehicular Technology Conference 515 (VTC Spring), 2016, pp. 1-5. doi:10.1109/VTCSpring.2016.7504361 [5] B. Di, L. Song, Y. Li, Radio resource allocation for uplink sparse code multiple access (scma) networks using matching game, in: 2016 IEEE International Conference on Communications (ICC), 2016, pp. 1-6. doi: 10.1109/ICC.2016.7511409

5. R. Dawadi, S. Parsaeefard, M. Derakhshani, T. Le-Ngoc, Power-efficient resource allocation in noma virtualized wireless networks, in: 2016 IEEE 570 Global Communications Conference (GLOBECOM), 2016, pp. 1-6. doi: 10.1109/GLOCOM.2016.7842162.

6. B. Li, Z. Bai, X. Zuo, Z. Yan, Y. Liang, Semi-granted sparse code multiple access (scma) for 5g networks, in: 2017 3rd EAI International Conference on IoT as a Service (IoTaaS 2017), 2017, pp. 1-8.

7. S. Zhang, B. Di, L. Song, Y. Li, Radio resource allocation for nonorthogonal multiple access (noma) relay network using matching game, in: 2016 IEEE International Conference on Communications (ICC), 2016, pp. 1-6. doi:10.1109/ICC.2016.7510918.

8. Z. Yuan, g. Yu, W. Li, S. Han, C. 1. I, Z. Wang, Multi-user shared access for 5g, Telecommun. Network Technology 5 (5) (2015) 28-30.

9. K. Au, L. Zhang, H. Nikopour, E. Yi, A. Bayesteh, U. Vilaipornsawai, J. Ma, P. Zhu, Uplink contention based scma for $5 \mathrm{~g}$ radio access, in: 2014 IEEE Globecom Workshops (GC Wkshps), 2014, pp. 900-905. doi:10. 1109/GLOCOMW.2014.7063547.

\section{AUTHORS PROFILE}

K.Raghavendra ,Assistant Professor,Dept. of ECE, Mallareddy Engineering College for women, Secunderabad, raghu.siiet@ gmail.com Dr. N.Sreekanth,Professor, Dept. of ECE, Mallareddy Engineering College for women, Secunderabad, nsreeku@ gmail.com

P.Swamy ,Assistant Professor, Dept.of ECE, TKREC,Ranga Reddy, swamygouds@gmail.com 